Canadian Oncology

Nursing Journal

Revue canadienne

de soins infirmiers

en oncologie

Volume 30, Issue 2 • Spring 2020

elSSN: 2368-8076 


\section{Changes in the uptake of screening for prostate cancer with prostate-specific antigen in Ontario between 2003 to 2012}

\section{by Louis Watson}

\section{ABSTRACT}

Objectives: This study investigates change in prostate-specific antigen (PSA) screening uptake in Ontario among men 35 years of age and older, between 2003 and 2012. We identify factors related to PSA testing, and examine the degree to which age guidelines for PSA testing are being followed or if PSA screening is opportunistic in nature. The relationship of income, education and having a regular physician and an estimation of PSA testing prevalence in 2012 is also studied.

Methods: Data from the Canadian Community Health Survey (CCHS) were used to estimate the proportion of men who had a PSA test in 2003 and then compared to a similar estimate (in similar population) in 2012. We also examined associations with PSA uptake to socio-demographic factors. Contingency table analyses were performed to characterize the changes; logistic regression was also used to evaluate the differences, while taking into account the possible confounding role of other factors.

Results: We found among men aged 35-49 years and 75+, there was an increase in PSA utilization between 2003 and 2012. In 2003, 18\% of men 35-49 years, and 74\% of men aged 75+ reported ever having a PSA test. These figures increased to $20 \%$ of men aged 35-49 years, and 84\% of men aged 75+ years in 2012. Logistic regression showed that a man in the 70-74 age category in 2012 had the highest likelihood of having had a PSA test compared to men in the age range of 35-49 years (odds ratio $(O R)=25$.9; confidence interval $(C I)=19.9-33.8)$.

Conclusion: In Ontario, PSA testing is seemingly opportunistic in nature and occurring in groups not recommended for testing by the Ontario guidelines. There is a marked increase in PSA utilization in age ranges outside of the current Ontario PSA testing guidelines (35-49 and 75+). It would appear that most PSA testing in Ontario is opportunistic and adherence to age guidelines is limited.

Keywords: cross-sectional study, Canada/Ontario; PSA test; prostate cancer; cancer screening

\section{AUTHOR NOTE}

Louis Watson, email: 14lw23@queensu.ca

DOI:10.5737/23688076302125132

\section{INTRODUCTION}

P rostate cancer (PC) is the most common cancer among Canadian men affecting more than 175,000 Canadians. An estimated 23,600 new cases will be diagnosed in Canada each year, including 9,600 in Ontario. The estimated five-year survival of men diagnosed with PC in Ontario is $97 \%$ (Drost et al., 2018). The current estimated economic burden of PC in Canada is unknown, but in 2000 the lifetime cost of PC was reported at totals of $\$ 9.76$ billion(Grover et al., 2000). After the introduction of prostate specific antigen (PSA) testing in the early 1990s, the rates of PC in Canada and United States of America (USA) became the highest in the world (Culp et al., 2019).

The PSA test is based on the fact that prostate-specific antigens are glycoproteins produced primarily by the prostate epithelial cells that line the ducts and acini of the prostate. It is thought that the disruption of the normal prostate glandular architecture facilitates the PSA's access to the systemic circulation, thus higher levels of PSA in a man's blood are indicative of possible PC or other prostate concerns (Kim \& Andriole, 2015). The PSA test was originally designed to measure the prostate's reaction to PC treatment. Following a study published in 1991, which showed that PSA testing was an effective method to screen for PC, PSA testing was approved in Canada (Catalona et al., 1991). The PSA test was later approved for use in the detection of PC in men with symptoms, but not for asymptomatic men (Kim \& Andriole, 2015). However, since 1991, PSA testing has been increasingly utilized as a screening tool to detect PC in men with no symptoms. Although there is no agreed value for what constitutes a 'normal' PSA value $<4.0 \mathrm{ug} / \mathrm{L}$ is generally considered normal, while a level $\geq 4.0 \mathrm{ug} / \mathrm{L}$ has been deemed to require further investigation (Taksler et al., 2018). However, it should be noted that the PSA test is not diagnostic. If the PSA is raised, a prostate biopsy is required to formally diagnose PC (Taksler et al., 2018).

Before the introduction of PSA testing in Canada, rates of PC were reported as $3.4 \%$ a year; these have increased to $25.4 \%$ following the introduction of PSA testing (Feletto et al., 2015). Given a positive PSA test requires further investigation in order to establish a malignancy, a false positive PSA result can lead to potentially unnecessary treatment such as a prostatic biopsy. The follow-up diagnostic tests also include potential harms such as psychological anxiety; post biopsy complications such as fever, pain, hematospermia/hematuria and sometimes sepsis; and many post-surgical complications (Fenton et al., 2018). The benefit of PSA screening is still controversial; evidence for mortality reduction from trials is 
conflicting and potential gains in life expectancy are opposed by losses in quality of life (QoL) due to over-diagnosis and overtreatment. Moreover, due to the uncertain benefit, medical organizations in Europe and the United States, including the European Association of Urology and the United States Preventive Services Task Force (USPSTF), do not recommend PSA testing/screening for any man (Ito et al., 2019).

In response to the USPSTF released recommendations, the Canadian Task Force on Preventive Health Care (CTFPH) followed with mirror recommendations against PSA screening for healthy men of any age (LeBlanc, Demers \& Shaw, 2019). In Ontario, the current guidelines for PSA testing for screening/ diagnosing purposes are as follows:

- Men aged 40 years or older with a family history of prostate cancer,

- Men racially at risk of prostate cancer, such as African-Canadians,

- Men aged 50 to 75 years old (who have a life expectancy of at least 10 years or more, however, Ontario guidelines do not recommended PSA screening for men over 75 years) and,

- Men at any age who have a physician willing to screen him, but must pay a fee (Webster, Lau, \& Newell, 2017).

Despite changes to the provincial and national guidelines in recent years, and the equivocal value of PSA testing, attempts to characterize changes in PSA screening uptakes at the general population level have been scarce. The objectives of this study are to quantify changes in PSA testing in Ontario males aged 35 or older from 2003 to 2012, and to study the association of selected factors, (i.e., age, income, education and having a regular physician) to the likelihood of PSA testing. We also examined the extent to which current PSA testing guidelines are being observed.

\section{MATERIAL AND METHODS}

\section{Study population}

The Canadian Community Health Survey (CCHS) is a cross-sectional survey that collects information related to health status, healthcare utilization and health determinants for the Canadian population. It relies upon a large sample of respondents and is designed to provide reliable estimates at the health region level. The CCHS was designed to cover approximately $98 \%$ of the non-institutionalized household population aged 12 years or older in all provinces and territories. In 2003, the CCHS used three sampling frames to select the sample of households. During this cycle, the CCHS surveyed 134,072 individuals nationally, including 42,777 within Ontario. Each respondent was from a separate household. The majority of the respondents resided within an area frame. In some health regions, a Random Digit Dialling (RDD) sampling frame or a list frame of telephone numbers was also used. The response rate for the 2003 CCHS was $80.7 \%$. A description of the CCHS survey is available at Statistics Canada's website (McAlpine et al., 2018).

For the 2012 CCHS data collection, 61,707 participants were interviewed across Canada and 21,257 within Ontario. Interviews were conducted using computer assisted personal interviewing, and most interviews (87\%) were conducted in person. The response rate was $79.8 \%$. Overall, there was a $53 \%$ decrease in sample size from 2003 to 2012 nationally and a $50 \%$ decrease for Ontario. Independent responding samples were interviewed for the 2003 and 2012 surveys. For our study, we used the public data micro file.

\section{Data collected}

For this study, respondents consisted of men residing in Ontario who were 35 years or older and provided data related to PSA screening. The study sample was categorized into five age groups, corresponding to the age guidelines for PSA testing, 35-49, 50-59, 60-69, 70-74, and 75+ years. Sociodemographic characteristics considered in the analysis were: age at interview, have a regular doctor, education, household income, smoking status, marital status, racial origin, and country of birth. Because income was categorized somewhat differently in each survey, we defined the income variable dichotomously as $<\$ 80,000$ and $\geq \$ 80,000$.

\section{Analytic strategy}

Analyses were restricted to Ontario men, 35 years of age and older, who responded to the question about having ever had a PSA blood test. Frequency estimates were produced to describe the characteristics of the study population. The data were weighted to represent the male population in Ontario aged 35-75+ using sample weights derived for the CCHS. The questions that followed the PSA test utilization included: time of last PSA test, reasons for PSA testing; family history, regular check-up, age, follow-up for problems/prostate problems, and other. The questions were identical in surveys for both years, with the exception of "race" as a possible reason for PSA testing, which was subsequently dropped from the analysis. P-values were only calculated for unadjusted variables and tested for significance at $95 \%$ confidence for the proportion between 2003 and 2012 using z-tests.

Differences in PSA testing uptake between 2003 and 2012 were quantified and categorized and differences among variables were evaluated using a Chi square test. Logistic regression models were then fitted in order to examine the influence of selected factors, including age, income and having a regular physician, on the likelihood of testing. To further investigate whether PSA screening is opportunistic in nature and what the main driving force behind opportunistic PSA screening is in Ontario, we ran a separate analysis. We created two new categories, 'opportunistic' and 'non-opportunistic', by using the Ontario PSA testing guidelines. A man was entered into the opportunistic category if he met the following criteria:

- PSA testing occurred in the absence of symptoms

- PSA testing occurred in the absence of family history or racial susceptibility

- PSA testing occurred in age groups 35-50 and 75+ years old

- Men who reported having had a PSA test within one year of survey participation.

Non opportunistic was defined as meeting the Ontario PSA guidelines and included men who reported having had a PSA test within one year of survey participation (Driedger et 
al., 2017). We then used the newly created variable (opportunistic yes/no) as a dichotomous dependent variable and fitted logistic regression models in order to examine the influence of selected factors on the likelihood of opportunistic and non-opportunistic PSA testing. All statistical analyses were performed on SAS statistical software, release 9.1 (Saab et al., 2018). The level of statistical significance was defined as $\mathrm{P}<0.05$.

\section{RESULTS}

The characteristics of men sampled by the CCHS in 2003 and 2012 do not differ significantly (see Table 1), although men in 2012 were noticeably older. In both years, more than $90 \%$ reported having a regular physician and the vast majority of those who had had PSA testing reported the test was included as part of a regular medical check-up. However, compliance with Ontario guidelines looks better in 2003 than 2012 (Figure 1). There was an increase in all age groups utilizing the PSA test from 2003 to 2012. PSA utilization as part of a regular medical check-up was identified as the most common reason for testing between 2003 and 2012. Both years were essentially unchanged for reported reasons as to having had a PSA test. In 2003 and 2012, greater than 50\% of men reported ever having a PSA test within one year (see Table 2).

In 2003, the associations of PSA test utilization with age was more pronounced among men at income levels equal to or greater than $\$ 80,000$ and who had access to a regular medical physician, compared with men in lower income levels. This effect was amplified in 2012 (Figure 3). The same relationships emerged when the analysis was restricted to men without a regular physician (Figure 4). After adjusting for age, logistic regression in 2012 found significant predictor variables for PSA testing: men who reported having a regular medical doctor were over four times more likely to have had a PSA test than men who do not have a regular medical doctor (OR 4.4 CI 3.7-4.9<0.05); men with a post-secondary education were significantly more likely to have had a PSA test compared with men who were less educated (OR 2.1 CI 1.8-2.4 $<0.05$ ), men with an income over $\$ 80,000$ (OR 1.6 CI 1.5-1.85), non-smokers (OR 1.7 CI 1.6-2.1), and being married (see table 3). Moreover, the strongest predictor of ever having had a PSA test in 2012 was the age group 70-75 years (OR 27.9 CI 22.133.9). Figure 2 displays PSA utilization by age compared to the age guidelines for PSA testing within Ontario, and shows in 2012 PSA testing is increasing in age groups more than 55 years old, as compared to 2003. Table 4 displays our analysis for opportunistic PSA testing in 2012. We found the strongest predictor for opportunistic and non-opportunistic PSA screening after adjustment was men who reported having a regular physician (OR 2.3 CI 1.2-4.4).

\section{DISCUSSION}

Overall, we found that PSA test utilization in Ontario increased over the period 2003 to 2012. The results of the analysis suggest that for the majority of men receiving testing it is part of a medical check-up, and they have a regular medical doctor. Advancing age is also strongly related to the likelihood of having had a PSA test. We concluded that PSA testing in
Ontario does appear to be opportunistic in nature. This is further illustrated by the fact that if physicians and patients were observing Ontario's guidelines for PC screening, then those reporting PSA testing would give the reason as being family history, age, prostate problem or general follow-up for a problem. This is not supported by the data, although no validation of the CCHS responses has been undertaken.

Why the effect of age, income and education were more significant in 2012 than 2003 may be explained by the participants being older in the 2012 sample, which would account for men being more educated. However, given the nature of a cross-sectional survey, it is difficult to explain certain nuances within the data. Attempting to explain the modifying effect of age, which was the strongest in 2012, especially when combined with income, may be that older and wealthier men are more likely to have physical and regular medical check-ups and, thus, have better access and more opportunity to discuss prostate cancer screening. An alternative explanation for the modifying effect of age is that, in 2012, younger men are more aware of the risks associated with PSA screening and may be less inclined to seek PSA testing, placing less importance on PSA test utilization than an older man does. Moreover, a physician may be less willing to discuss PC screening with a younger man. Access to a physician may be a barrier for a younger man in full-time employment.

Overall, with our investigation into age and income, our logistical model showed that a man with an income $\geq \$ 80,000$ was much more likely to have had a PSA test with each increase in age category than a man who earns $<\$ 80,000$ with each increasing age category. Age is the primary driving factor in 2012 for PSA test utilization, although, all independent variables were much stronger predictors in 2012 than in 2003. The influence of a regular physician may not be as important as how old or wealthy a man is. Once PSA testing was categorized as opportunistic, age and having a regular physician were the only significant factors involved in predicting the likelihood of PSA testing.

The debate surrounding PSA testing centres on the fact that some men with potentially aggressive tumours will benefit from PSA testing and, age dependent, may benefit from treatment. However, some PC will be slow growing and may never cause any symptoms or shorten life expectancy and, in the absence of PSA testing, would never be picked up. Some men who are tested may, therefore, face unnecessary anxiety, medical tests, and treatments with side effects. How the recent CTFPH guidelines, which state that no man should be screened for PC regardless of age or risk factors, will influence Ontario's PSA testing guidelines is yet to be seen.

\section{IMPLICATIONS FOR PRACTICE}

Studies suggest that men are passive information gatherers when seeking PC screening, rather than active information seekers. Key sources of information included the Internet for active information-seekers and their family doctor and healthcare professionals for passive information-gatherers (Beaulac, Fry \& Onysko, 2006; Driedger et al., 2017; Jamies et al., 2017; McAlpine et al., 2018; Saab et al., 2018). Nurses are in a unique 


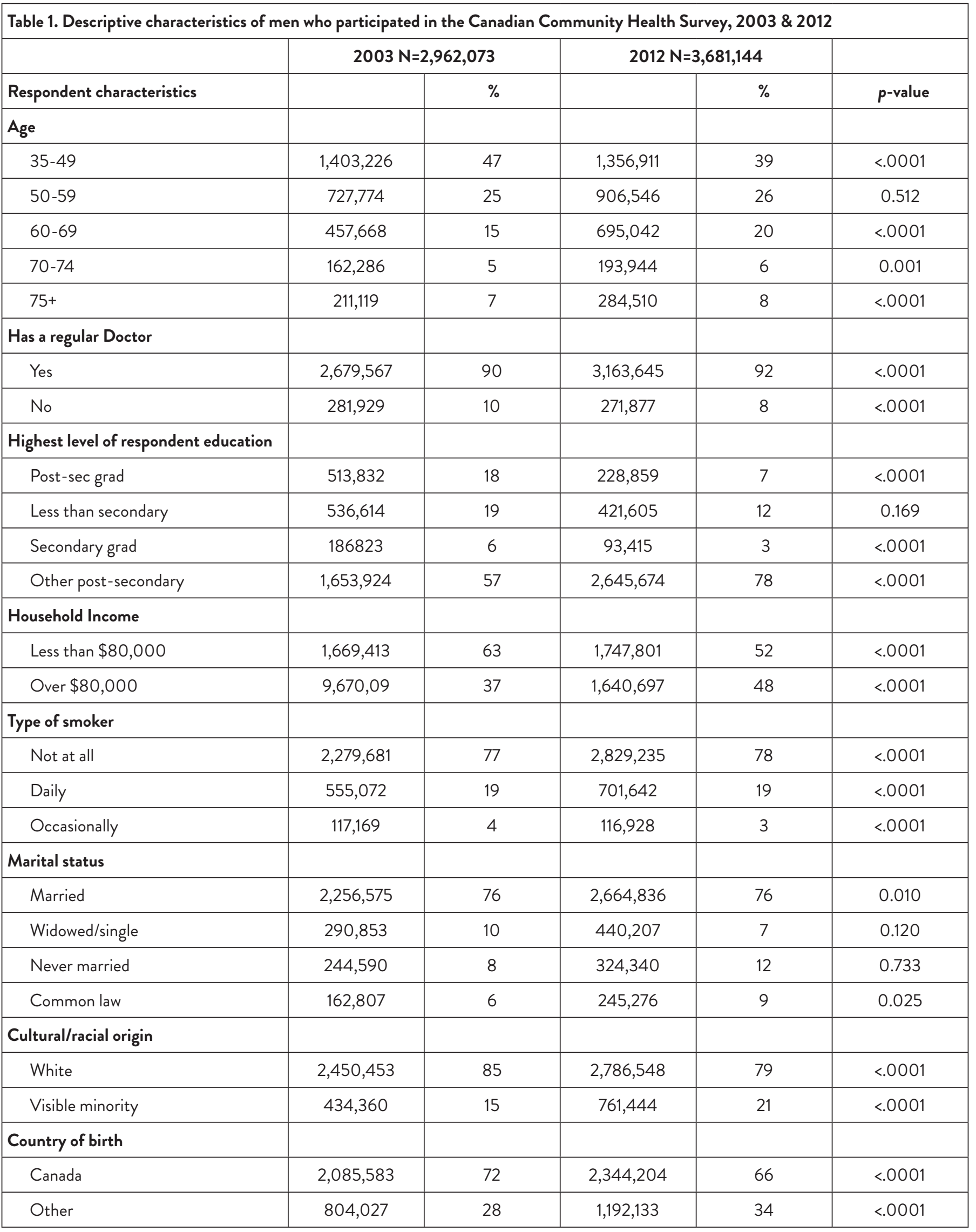




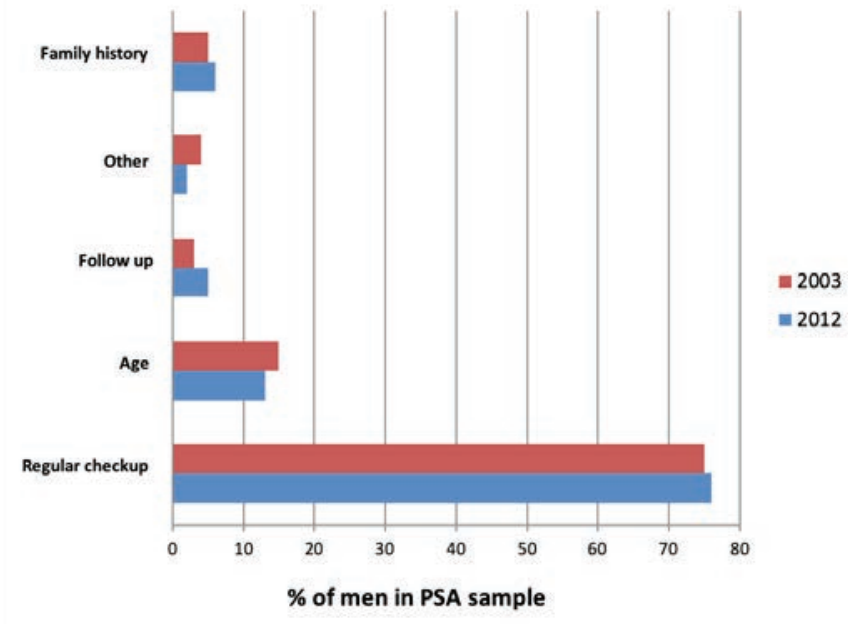

Figure 1. Percent of men (35+ years) who self-reported reasons for having had a PSA test among the 6,654 Ontario participants in 2003 and the 6,220 participants in 2012

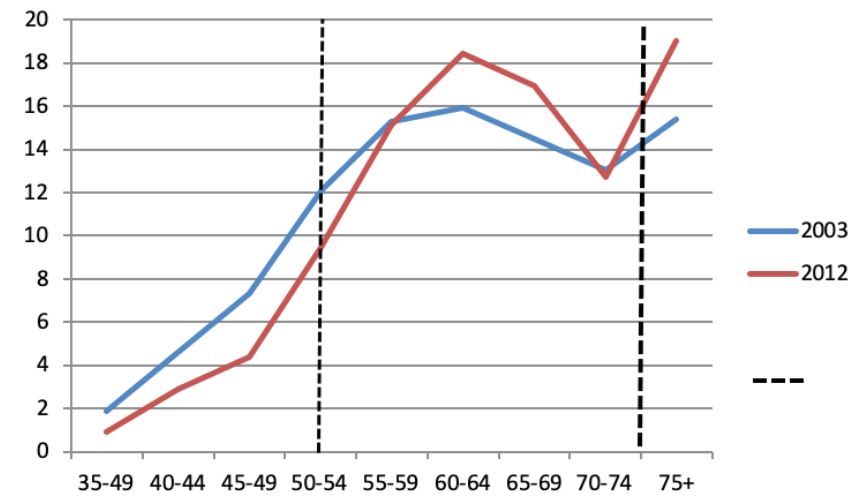

Figure 2. Number of men who self-reported having had a PSA test within the Ontario age guidelines (50-75 years old), and who participated in the Canadian Community Health Surveys 2003 and 2012

Represents 2012 Ontario PSA screening age guidelines

\begin{tabular}{|c|c|c|c|c|c|c|c|c|c|}
\hline & \multicolumn{4}{|c|}{$\mathrm{N}=2,962,072(2003)$} & \multicolumn{4}{|c|}{$N=3,681,144(2012)$} & \multirow[b]{3}{*}{$p$-value* } \\
\hline & \multicolumn{4}{|c|}{ Age group $\%$} & \multicolumn{4}{|c|}{ Age group $\%$} & \\
\hline & $35-49$ & $50-75$ & $75+$ & Overall \% & $35-49$ & $50-75$ & $75+$ & Overall \% & \\
\hline \multicolumn{10}{|c|}{ Have you ever had a PSA test? } \\
\hline Yes & 18 & 68 & 74 & 44 & 20 & 71 & 84 & 52 & $<0.001$ \\
\hline No & 82 & 32 & 26 & 56 & 80 & 29 & 16 & 48 & \\
\hline \multicolumn{10}{|c|}{ When was the last time you had a PSA test? } \\
\hline$\leq 1$ year & 17 & 72 & 11 & 58 & 15 & 74 & 11 & 55 & $<0.001$ \\
\hline$>1$ year $=83$ & 28 & 89 & 42 & 85 & 26 & 89 & 45 & & \\
\hline \multicolumn{10}{|c|}{ Reasons for PSA test from men who had a test } \\
\hline & \multicolumn{4}{|c|}{$n=1,261,321(2003)$} & \multicolumn{4}{|c|}{$n=1,923,239(2012)$} & \\
\hline \multicolumn{10}{|c|}{ Part of regular check up } \\
\hline Yes & 75 & 78 & 67 & 76 & 75 & 77 & 72 & 76 & $<0.001$ \\
\hline No & 25 & 22 & 33 & 24 & 25 & 23 & 28 & 24 & \\
\hline \multicolumn{10}{|l|}{ PSA for age } \\
\hline Yes & 8 & 9 & 18 & 15 & 75 & 77 & 72 & 24 & $<0.001$ \\
\hline No & 92 & 91 & 82 & 85 & 15 & 23 & 28 & 76 & \\
\hline \multicolumn{10}{|c|}{ PSA for follow up problem } \\
\hline Yes & 8 & 9 & 18 & 10 & 6 & 9 & 18 & 10 & $<0.001$ \\
\hline No & 92 & 91 & 82 & 90 & 4 & 91 & 82 & 90 & \\
\hline \multicolumn{10}{|c|}{ PSA follow-up prostate treatment } \\
\hline Yes & 1 & 3 & 10 & 3 & 0 & 3 & 11 & 4 & $<0.001$ \\
\hline No & 99 & 77 & 90 & 97 & 100 & 77 & 89 & 96 & \\
\hline \multicolumn{10}{|l|}{ PSA other } \\
\hline Yes & 4 & 4 & 4 & 4 & 6 & 9 & 18 & 10 & 0.359 \\
\hline No & 96 & 96 & 96 & 96 & 94 & 91 & 82 & 90 & \\
\hline \multicolumn{10}{|c|}{ PSA family history } \\
\hline Yes & 11 & 4 & 3 & 5 & 14 & 6 & 2 & 6 & 0.195 \\
\hline No & 89 & 96 & 97 & 95 & 86 & 94 & 98 & 94 & \\
\hline
\end{tabular}




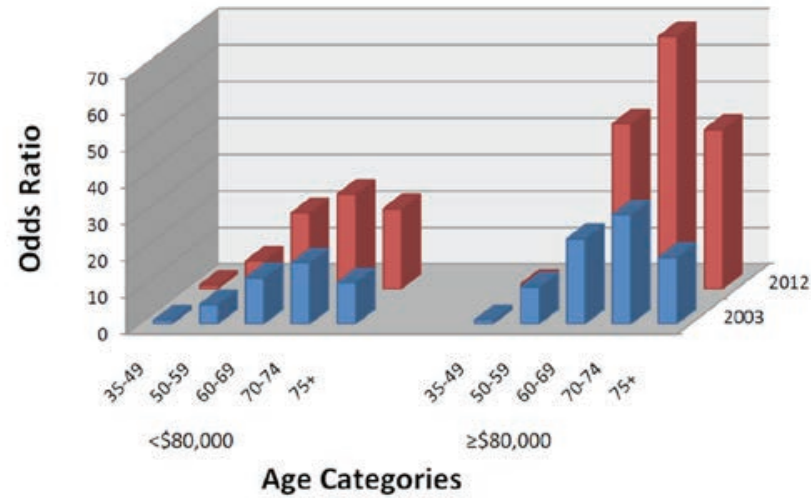

Figure 3. Bar chart showing the relationship between having a regular medical physician, age and income on likelihood of ever having had a PSA test for 2003 and 2012

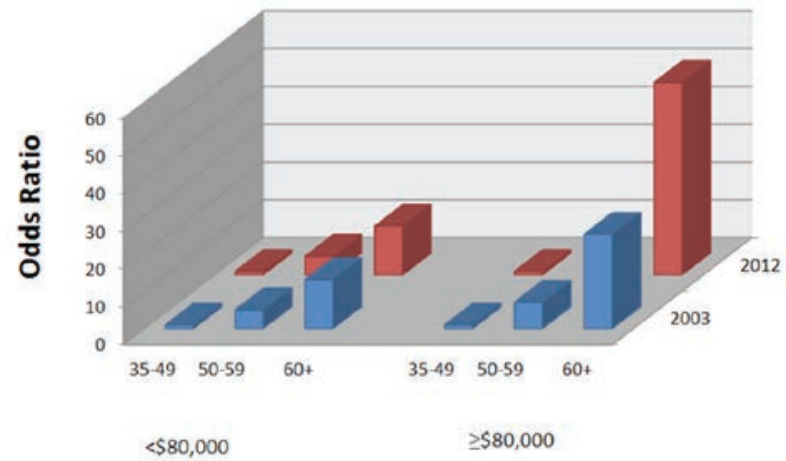

Age Categories

Figure 4. Bar chart showing the relationship between not having a regular physician, age and income on likelihood of ever having has a PSA test for 2003 and 2012

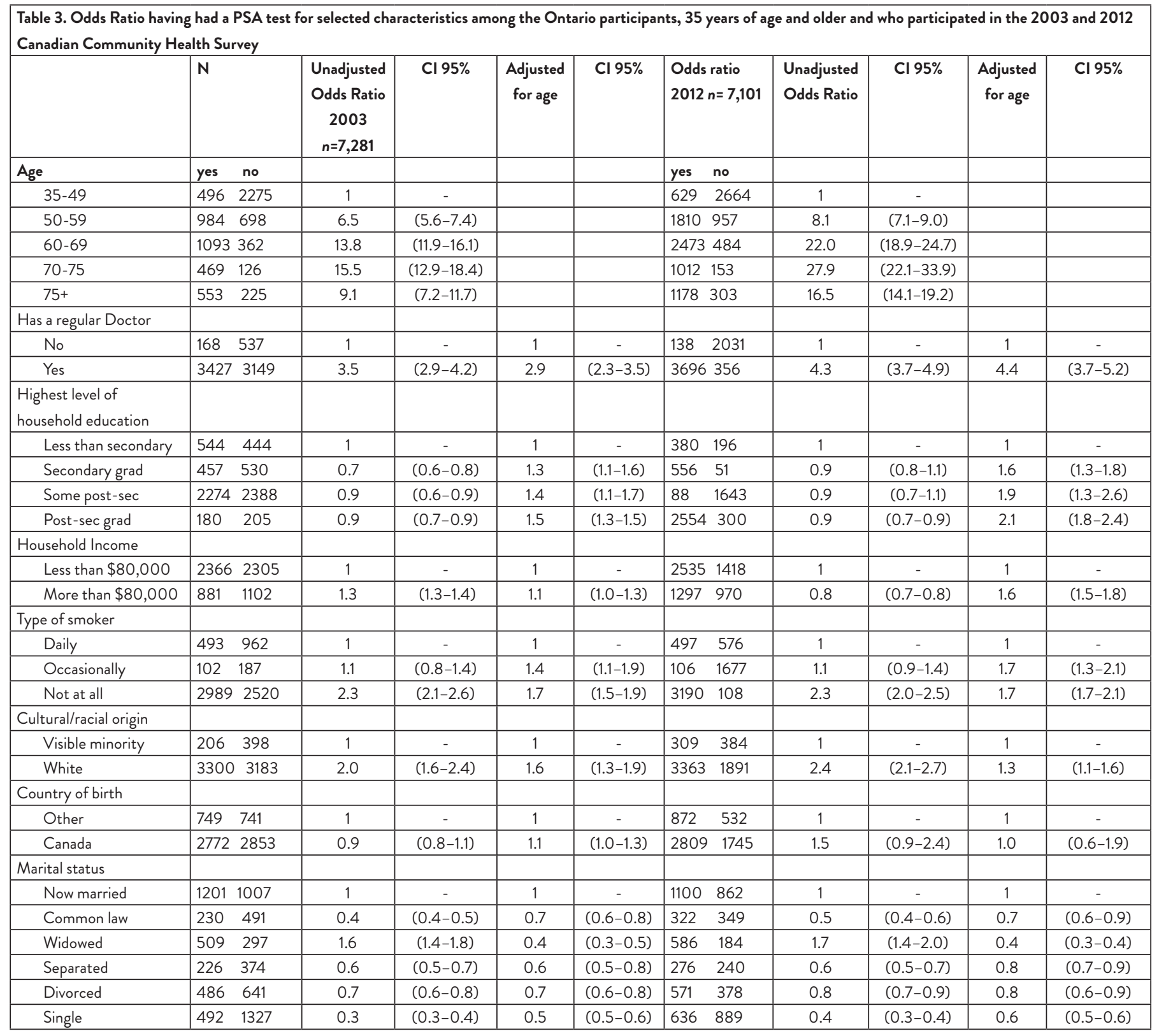


Table 4. Opportunistic versus Non-Opportunistic Comparison

\begin{tabular}{|c|c|c|c|c|c|c|c|}
\hline \multirow[t]{2}{*}{ PSA <1 year* } & \multirow{2}{*}{\begin{tabular}{|l|} 
Oppt \\
$n$
\end{tabular}} & \multirow{2}{*}{$\begin{array}{l}\text { NonOpp }+\dagger \\
n\end{array}$} & \multirow[b]{2}{*}{$\% * *$} & \multicolumn{2}{|l|}{ Unadjusted } & \multicolumn{2}{|l|}{ Adjusted \pm} \\
\hline & & & & Odds ratio & $\mathrm{Cl} 95 \%$ & Odds ratio & $\mathrm{Cl} 95 \%$ \\
\hline \multicolumn{8}{|c|}{ Has a regular medical doctor } \\
\hline Yes & 913 & 2880 & 31 & 1.9 & $1.1-3.4$ & 2.3 & $1.2-4.4$ \\
\hline No & 14 & 84 & 17 & 1 & - & 1 & - \\
\hline \multicolumn{8}{|l|}{ Education } \\
\hline Less than secondary & 133 & 237 & 56 & 1 & - & 1 & - \\
\hline Secondary grad & 134 & 383 & 35 & 0.6 & $0.5-0.8$ & 0.7 & $0.6-1.0$ \\
\hline Other post-sec & 17 & 78 & 22 & 0.4 & $0.2-0.7$ & 0.5 & $0.3-0.9$ \\
\hline Post-sec grad & 588 & 2056 & 29 & 0.5 & $0.4-0.6$ & 0.7 & $0.5-0.9$ \\
\hline \multicolumn{8}{|l|}{ Household Income } \\
\hline$<\$ 80,000$ & 637 & 1825 & 35 & 1 & - & 1 & - \\
\hline$\geq \$ 80,000$ & 290 & 1086 & 27 & 0.8 & $0.7-0.8$ & 1.1 & $1.0-1.7$ \\
\hline \multicolumn{8}{|l|}{ Type of smoker } \\
\hline Daily & 65 & 348 & 16 & 1 & - & 1 & - \\
\hline Occasionally & 24 & 70 & 26 & 1.8 & $1.1-3.1$ & 1.8 & $1.1-3.3$ \\
\hline Not at all & 823 & 2477 & 25 & 1.8 & $1.3-2.3$ & 1.6 & $1.2-2.1$ \\
\hline \multicolumn{8}{|l|}{ Marital status } \\
\hline Single/never married & 99 & 212 & 20 & 1 & - & 1 & - \\
\hline Married & 563 & 2047 & 13 & 0.6 & $0.5-0.8$ & 0.5 & $0.3-0.6$ \\
\hline Common-law & 34 & 131 & 11 & 0.6 & $0.4-0.9$ & 0.5 & $0.3-0.8$ \\
\hline Widowed & 157 & 149 & 36 & 2.3 & $1.6-3.1$ & 1.3 & $0.9-1.9$ \\
\hline Separated & 28 & 118 & 11 & 0.5 & $0.3-0.8$ & 0.5 & $0.3-0.8$ \\
\hline Divorced & 46 & 248 & 6 & 0.4 & $0.3-0.6$ & 0.4 & $0.2-0.5$ \\
\hline \multicolumn{8}{|l|}{ Immigrant } \\
\hline Yes & 240 & 636 & & 1 & & 1 & - \\
\hline No & 653 & 2200 & & 1.3 & $1.1-1.5$ & 1.0 & $0.5-2.1$ \\
\hline
\end{tabular}

*527 men answered "Don't know" or "refused to answer" to the inclusion criteria questions, such as PSA for family history were dropped from the analysis.

†Opp $=$ Opportunistic PSA screening, defined as, under 50 years old, over 75 years old, had PSA for asymptomatic screening, was not reported as black or African origin and had a PSA in within the last year.

$\dagger †$ Nonopp=Non opportunistic PSA screening, defined as meeting the Ontario PSA testing guidelines, i.e, 50-75 years old, and all races.

**Percentage is presented as a proportion between opportunistic and non-opportunistic PSA screening for each category

\pm Adjusted for age, education, household income, type of smoker, marital status and immigrant 
position to communicate the risks and benefits of PC screening in a way patients can understand. Studies such as ours can be used to understand the motivation behind seeking PC screening and may be influenced by multiple factors including, access to a doctor, age, education and income. Nurses can educate patients on the harms and benefits of PC screening and mobilize knowledge such as the recent USPSTF PC screening recommendations (i.e., no man to have a PSA test), along with the Ontario guidelines. Further, nurses can inform a patient regarding arguments supporting PSA testing (e.g., malignancies can be caught early and potentially save lives) and the harms (e.g., a false positive PSA test can lead to unnecessary treatments and exposure to risks, such as infections).

\section{REFERENCES}

Beaulac, J. A., Fry, R. N., \& Onysko, J. (2006). Lifetime and recent prostate specific antigen (PSA) screening of men for prostate cancer in Canada. Canadian Journal of Public Health, 97(3), 171-176.

Catalona, W. J., Smith, D. S., Ratliff, T. L., Dodds, K. M., Coplen, D. E., Yuan, J. J., Petros, J. A., \& Andriole, G. L. (1991). Measurement of prostate-specific antigen in serum as a screening test for prostate cancer. New England Journal of Medicine, 324(17), 1156-1161.

Culp, M. B., Soerjomataram, I., Efstathiou, J. A., Bray, F., \& Jemal, A. (2019). Recent global patterns in prostate cancer incidence and mortality rates. European Urology. https://www.ncbi.nlm.nih.gov

Driedger, S. M., Annable, G., Brouwers, M., Turner, D., \& Maier, R. (2017). Can you un-ring the bell? A qualitative study of how affect influences cancer screening decisions. BMC Cancer, 17(1), 647.

Drost, F.-J. H., Rannikko, A., Valdagni, R., Pickles, T., Kakehi, Y., Remmers, S., van der Poel, H. G., Bangma, C. H., \& Roobol, M. J. (2018). Can active surveillance really reduce the harms of overdiagnosing prostate cancer? A reflection of real life clinical practice in the PRIAS study. Translational Andrology and Urology, 7(1), 98.

Feletto, E., Bang, A., Cole-Clark, D., Chalasani, V., Rasiah, K., \& Smith, D. P. (2015). An examination of prostate cancer trends in Australia, England, Canada and USA: Is the Australian death rate too high? World Journal of Urology, 33(11), 1677-1687.

Fenton, J. J., Weyrich, M. S., Durbin, S., Liu, Y., Bang, H., \& Melnikow, J. (2018). Prostate-specific antigen-based screening for prostate cancer: A systematic evidence review for the US preventive services task force. JAMA, 319(18), 1914-1931. https:// doi.org/10.1001/jama.2018.3712

Grover, S.A., Coupal, L., Zowall, H., Rajan, R., Trachtenberg, J., Elhilali, M., Chetner, M., \& Goldenberg, L. (2000). The economic burden of prostate cancer in Canada: Forecasts from the Montreal Prostate Cancer Model. CMAJ, 162(7), 987-992.

\section{CONCLUSION}

In the absence of a clear interpretation of our results, and due to the cross-sectional nature of the data, causality between related factors and testing cannot be inferred. However, our findings do suggest that adherence to Ontario PSA testing guidelines with regard to age is low, and physician-driven opportunistic PSA screening is prevalent. Moreover, when considering the risks of false positives and physical harm due to prostate cancer screening, along with the economic burden of over-diagnosis, this study's results should be at the forefront of any new research or policy revision within Ontario.

\section{ACKNOWLEDGEMENTS}

Special thanks to Dr. Paul Villeneuve, as without his expert guidance and support, this study would not have been possible.

Ito, K., Oki, R., Sekine, Y., Arai, S., Miyazawa, Y., Shibata, Y., Suzuki, K., \& Kurosawa, I. (2019). Screening for prostate cancer: History, evidence, controversies and future perspectives toward individualized screening. International Journal of Urology, 26(10), 956-70. https://doi.org/10.1111/iju.14039

James, L. J., Wong, G., Craig, J. C., Hanson, C. S., Ju, A., Howard, K., Usherwood, T., Lau, H., \& Tong, A. (2017). Men's perspectives of prostate cancer screening: A systematic review of qualitative studies. PloS one, 12(11), e0188258.

Kim, E. H. \& Andriole, G. L. (2015). Prostate-specific antigen-based screening: Controversy and guidelines. BMC Medicine, 13(1), 61.

LeBlanc, A. G., Demers, A., \& Shaw, A. (2019). Recent trends in prostate cancer in Canada. Health Reports, 30(4), 12-17.

Light, A., Elhage, O., Marconi, L., \& Dasgupta, P. (2019). Prostate cancer screening: Where are we now? BJU International, 123(6), 916-917.

McAlpine, K., Lewis, K. B., Trevena, L. J., \& Stacey, D. (2018). What is the effectiveness of patient decision aids for cancer-related decisions? A systematic review subanalysis. JCO Clinical Cancer Informatics, 2, 1-13.

Saab, M. M., Reidy, M., Hegarty, J., O'Mahony, M., Murphy, M., Von Wagner, C., Drummond, F. J. (2018). Men's information-seeking behavior regarding cancer risk and screening: A meta-narrative systematic review. Psycho-oncology, 27(2), 410-419.

Taksler, G. B., Keating, N. L., \& Rothberg, M. B. (2018). Implications of false-positive results for future cancer screenings. Cancer, 124(11), 2390-2398.

Webster, T. M., Lau, E., \& Newell, K. J (2017). Implications of prostatespecific antigen screening guidelines on clinical practice at a Canadian regional community hospital. Canadian Urological Association Journal, 11(8), 283. 